ups, as well as attend patients that had undergone an oncological process via telephone. Due to their vulnerability, the psychological impact on this patient has been even worse than on other users of the health system. The aim in this study is to evaluate the concerns, global health status and quality of life of patients with gynecological cancer during the pandemic.

Methodology The GineonCoVID study is a multicenter Spanish study that collect data from a national survey. The anonymous survey consists of 23 questions regarding the personal experience of the patient and modifications in health care during follow up of patient with gynecological malignancies from April to May 2021. The survey has been divided into 3 sections. In this sub-analysis the results of the questions related to anxiety, concerns, quality of life and state of mind of the interviewed patients will be evaluated.

Result(s)* 376 patients responded to the survey. The median age was 58 years. $43 \%$ of the patients were diagnosed with endometrial cancer, $27.3 \%$ with ovarian cancer and $24.1 \%$ with cervical cancer. $39.6 \%$ and $33.6 \%$ of patients suffered anxiety and depression respectively during the pandemic. $81 \%$ of the patients report having a good quality of life during the last year. Results showed that $54.2 \%$ of the respondents are not concerns about viral infection and $67.9 \%$ consider that the risk of being infected during follow-up is low. $94 \%$ do not perceive changes in follow up, but if they do, $44 \%$ believe that clinical health care has experienced a change. $71.4 \%$ of the patients are concern about not being able to attend clinical visits.

Conclusion* The pandemic could increase anxiety and depression, although it does not appear to worsen the quality of life in patients with gynecological cancer. More than half of the patients consider that they have a low risk of being infected during follow-up but are concerned about not being able to attend clinical visits due to the pandemic.

\section{IMPACT OF COVID-19 PANDEMIC ON GYNECOLOGICAL CANCER MANAGEMENT: NATIONAL SURVEY. GINEONCOVIDSURG STUDY}

M Gracia*, MD Diestro, Y Pérez, V García, J Siegrist Ridruejo, I Zapardiel, A La Paz. University Hospital, Madrid, Spain

\subsection{6/ijgc-2021-ESG0.660}

Introduction/Background* The current COVID 19 pandemic is having a major impact on healthcare systems around the world. Modifications in health resources have been necessary to face this new situation, which have had a direct impact on clinical practice, also affecting the field of gynecology oncology. To date, the evidence of the changes that COVID-19 has implied in the management of patients with gynecological cancer is scarce.

The objective of this study is to evaluate the impact of the COVID-19 pandemic on the management of patients with gynecological cancer by a nationwide survey to oncogynecologists. Methodology Observational study that collects data from a national survey sent via email to Spanish oncogynecological surgeons on June 2021. The survey included a total of 35 questions which were divided into 6 sections including assessment of the personal characteristics of responders, questions related to the triage procedures and protective measures adopted in the hospital against COVID-19 and questions related to changes in the management of ovarian, endometrial and cervical cancer after COVID-19 outbreak.

Result(s)* Overall, 75 participants completed the questionnaire. The results showed that $85 \%$ of gynecologic surgeons were not concerned about COVID-19 outbreak and 79.7\% of the participants stated that COVID-19 has not significantly modified their everyday practice. All surgeons declared triage method adopted in order to minimise COVID 19 diffusion. $87.5 \%$ did not modify surgical approach, although $6.9 \%$ decreased laparoscopic use in favor of laparotomic surgery. Only $45.1 \%$ of the surgeons adopted specific protection against COVID-19 in robotic or laparoscopic surgery, however $55.6 \%$ of the participants adopted it in open surgery. The use of neoadjuvant chemotherapy increased in $31.9 \%$ in favor of primary cytoreduction in ovarian cancer. $11.3 \%$ and $15.5 \%$ of patient with cervical and endometrial cancer respectively suffered a delay in cancer treatment during the pandemic.

Conclusion* The conclusions of the study showed how the COVID-19 pandemic has influenced the surgical management of patients with gynecological cancer in different Spanish hospitals.

\section{Ovarian cancer}

\section{RESULTS OF THE AVOIDING LATE DIAGNOSIS OF OVARIAN CANCER (ALDO) PROJECT; A PILOT NATIONAL SURVEILLANCE PROGRAM FOR BRCA MUTATION- CARRIERS}

${ }^{1} \mathrm{~S}$ Philpott, ${ }^{2} \mathrm{M}$ Raikou, ${ }^{3} \mathrm{R}$ Manchanda, ${ }^{4} \mathrm{G}$ Evans, ${ }^{4} \mathrm{R}$ Edmondson, ${ }^{5} \mathrm{U}$ Menon, ${ }^{6} \mathrm{M}$ Ahmed, ${ }^{4} \mathrm{E}$ Woodward, ${ }^{7} \mathrm{~A}$ Lamnisos, ${ }^{1} \mathrm{M}$ Lockley, ${ }^{1} \mathrm{M}$ Scott, ${ }^{1} \mathrm{~N}$ Singh, ${ }^{8} \mathrm{~S}$ Skates, ${ }^{2} \mathrm{~A}$ Mcguire, ${ }^{1}$ A Rosenthal ${ }^{*} .{ }^{1}$ University College London Hospitals NHS Foundation Trust; ${ }^{2}$ London School of Economics; ${ }^{3}$ Barts Cancer Institute; ${ }^{4}$ Manchester University NHS Foundation Trust; ${ }^{5} \mathrm{MRC}$ Clinical Trials Unit, UCL; ${ }^{6}$ Great Ormond Street Hospital; ${ }^{7}$ The Eve Appeal, UK; ${ }^{8}$ Massachusetts General Hospital, Boston, USA

\subsection{6/ijgc-2021-ESG0.661}

Introduction/Background* Ovarian cancer (OC) in BRCA mutation-carriers is typically diagnosed clinically at $>=$ stage $3 \mathrm{c}$, with consequent poor prognosis. Risk-reducing salpingooophorectomy (RRSO) is recommended for BRCA mutationcarriers as the only proven method of OC prevention. Women who defer RRSO to permit child-bearing/prevent premature menopause would benefit from surveillance which can downstage OC occurring prior to RRSO. We wanted to establish the 'real world' performance of OC surveillance which we have previously shown downstages OC in clinical trials.

Methodology 875 female BRCA mutation-carriers were recruited at $13 \mathrm{UK}$ centres and via a media campaign and underwent 4-monthly surveillance with the Risk of Ovarian Cancer Algorithm (ROCA) blood test. They had a 6 week repeat test if their ROCA score was $>1$ in 1000, and a transvaginal scan (TVS) in addition, if their risk was $>1$ in 500. Women with a score $>1$ in 33 or those with concerning TVS were referred to a rapid access clinic to rule out OC. RRSO was encouraged throughout the program. Participants were followed via questionnaires, notification by centres/GPs and direct contact. Surveillance performance was calculated after censoring 4 months after prior screen, with modelling of occult cancers detected at RRSO. Incremental cost-effectiveness was calculated using a Markov population cohort simulation. 